Article

\title{
The Seebeck Coefficient of Sputter Deposited Metallic Thin Films: The Role of Process Conditions
}

\author{
Florian G. Cougnon and Diederik Depla *ii \\ Department of Solid State Sciences, Ghent University, Krijgslaan 281 (S1), 9000 Gent, Belgium; \\ florian.cougnon@ugent.be \\ * Correspondence: Diederik.Depla@ugent.be
}

Received: 9 April 2019; Accepted: 26 April 2019; Published: 1 May 2019

\begin{abstract}
Because of their reduced dimensions and mass, thin film thermocouples are a promising candidate for embedded sensors in composite materials, especially for application in lightweight and smart structures. The sensitivity of the thin film thermocouple depends however on the process conditions during deposition. In this work, the influence of the discharge current and residual gas impurities on the Seebeck coefficient is experimentally investigated for sputter deposited copper and constantan thin films. The influence of the layer thickness on the film Seebeck coefficient is also discussed. Our observations indicate that both a decreasing discharge current or an increasing background pressure results in a growing deviation of the film Seebeck coefficient compared to its bulk value. Variations in discharge current or background pressure are linked as they both induce a variation in the ratio between the impurity flux to metal flux towards the growing film. This latter parameter is considered a quantitative measure for the background residual gas incorporation in the film and is known to act as a grain refiner. The observed results emphasize the importance of the domain size on the Seebeck coefficient of metallic thin films.
\end{abstract}

Keywords: Seebeck coefficient; background pressure; impurities; discharge current; domain size; layer thickness; sputter deposition

\section{Introduction}

Thin film sensing applications, such as thin film thermocouples, can be very attractive for lightweight structures, small devices or applications in need of a high temporal, or spatial resolution as they have a very low mass, reduced dimensions, and a very fast response time [1-5]. For example, thin film thermocouples are used for nanoscale thermometry [6-8], for monitoring local temperature distributions on integrated-circuits [9,10], in solid oxide fuel cells [11], and turbine engines [12], or for monitoring sudden temperature changes in cutting tools for machining explosive materials [13]. Furthermore, thin film sensors can be embedded inside composite materials without affecting the structural integrity of the material by their dimensional extent, enabling local and in situ sensing without compromise. In the case of sensing applications, typically the circuit does not draw current but an open-gate voltage is measured and linked to a physical property. Studying the behavior of the Seebeck coefficient of thin films and the relation to the deposition process is therefore an interesting field. The Seebeck effect describes the observation of an induced voltage difference when a temperature difference is applied over a metal or semiconductor. The generated voltage scales linearly to the applied temperature difference and the proportionality factor is referred to as the Seebeck coefficient $S$.

Thermoelectric energy is transported by mobile charge carriers [14]. Typically, metals have a high charge carrier concentration and thus a low Seebeck coefficient but a high electrical conductivity, whereas semiconductors have a low charge carrier concentration and thus a high Seebeck coefficient and low electrical conductivity. For thermoelectric applications, i.e., the conversion of heat into 
electrical power, it is important to combine of a low thermal conductivity (increasing temperature difference), a high Seebeck coefficient (increasing thermoelectric voltage), and a high electrical conductivity (decreasing Ohmic losses). The interplay between these three material properties is summarized under a single parameter $Z=S \sigma^{2} / \kappa$, called the figure-of-merit. Presently, around half of the thermoelectric research and development is focused on the maximization of the figure of merit $\mathrm{Z}$ for semiconductor materials such as $\mathrm{PbTe}, \mathrm{Bi}_{2} \mathrm{Te}_{3}$ and $\mathrm{SiGe}$ [15]. Although semiconductors are expected to have higher Seebeck coefficients than metals, these materials are more expensive, have a more complex charge carrier transport mechanism and can quite often only be RF-sputter deposited which hinders to upscale the obtained results to industrial applications. Therefore, we solely study the behavior of the Seebeck coefficient for DC sputtered metallic thin films from a fundamental point of view. Despite the influence of layer thickness on the Seebeck coefficient of metallic films was already studied in the past [16-23], there is still much to be explored. In this study, we investigate the role of the process conditions, i.e., the discharge current and the background pressure, on the Seebeck coefficient of thin metallic films.

\section{Materials and Methods}

The samples discussed in the scope of this work are deposited in a cuboid stainless steel vacuum chamber with a volume of $0.7 \times 0.62 \times 0.56 \mathrm{~m}^{3}$. The films are grown on acetone-cleaned glass substrates (VWR International, Radnor, PA, USA) for the Seebeck measurements or either on RCA-cleaned silicon wafers in the case of film characterization. The samples were measured by $\mathrm{X}$-ray diffraction (Bruker D8, Billerica, MA, USA) in a Bragg-Brentano configuration with a parallel beam bundle defined by the PolyCap. The peaks in the diffractogram were fitted to a Lorentzian-shaped curve and the Debye-Scherrer equation was used to determine the domain sizes. During all depositions, the pumping speed was kept constant $(\approx 75 \mathrm{l} / \mathrm{s})$ and the substrate was grounded and not externally heated or cooled. The used copper and constantan sputter targets are two inch circular planar targets (purity 99.99\%, Testbourne, Hampshire, UK). The films deposited for Seebeck measurements are patterned using a sputter mask specified in Figure 1. After deposition, a complementary bulk wire is attached to the thin film in order to form a conventional E-type, i.e., $\mathrm{Cu}_{55} \mathrm{Ni}_{45}(\mathrm{film})+$ $\mathrm{Ni}_{90} \mathrm{Cr}_{10}$ (wire), or T-type thermocouple, i.e., $\mathrm{Cu}$ (film) $+\mathrm{Cu}_{55} \mathrm{Ni}_{45}$ (wire). The $\mathrm{Cu}_{55} \mathrm{Ni}_{45}$ and $\mathrm{Ni}_{90} \mathrm{Cr}_{10}$ wire (Goodfellow, Cambridgeshire, UK) had a diameter of $0.125 \mathrm{~mm}$ and a polyimide insulation. The Seebeck measurements are performed with a home-built system. The setup consists of a heating element (MeiVac substrate heater, San Jose, CA, USA) and a water-cooled copper block, separated over a distance of $\approx 8 \mathrm{~cm}$. The bimetallic film-wire junction of the sample is centered on the heating element, whereas the electrodes are centered on the copper block. Thermal paste (Dow Corning, Midland, MI, USA) was applied at both ends of the glass substrate in order to enhance the heating or cooling transfer. The water-cooled copper block is kept at a constant temperature of $11^{\circ} \mathrm{C}$. The heating element is PID-controlled and ramps from room temperature to $60^{\circ} \mathrm{C}$ at a rate of $1{ }^{\circ} \mathrm{C} / \mathrm{min}$. Due to the imposed temperature difference, an open-circuit thermoelectric voltage arises at the electrodes of the film-wire thermocouple. The output of the thermocouples was logged by means of a TC01 (National Instruments, Austin, TX, USA), a thermocouple measurement device with built-in software for data acquisition. In order to provide a good electrical contact with the film for the read-out, a fine copper brush was clamped onto the thin film. The hot junction and cold junction temperature were measured by means of a K-type thermocouple. A Labview code managed all the data and plotted the measured voltage as function of the temperature difference between the hot and cold junction. A straight line was fitted to the data ( $\approx 200$ measurement points) and the slope of this line was used to determine the Seebeck coefficient. The experimental values for the E- and T-type bulk thermocouples (i.e., wire + wire) are used as reference and are $(67.3 \pm 1.4) \mu \mathrm{V} /{ }^{\circ} \mathrm{C}$ and $(46.3 \pm 0.8) \mu \mathrm{V} /{ }^{\circ} \mathrm{C}$ respectively. 


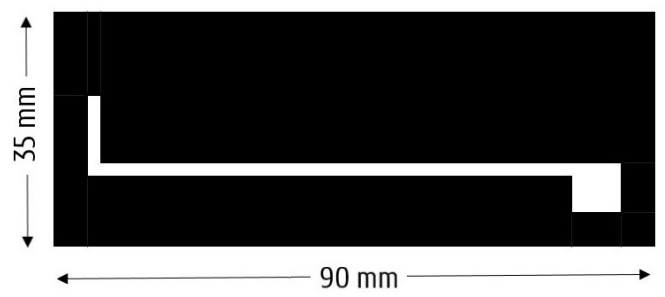

Figure 1. Design of the sputter mask used for pattering the thermocouple leg. The mask is fabricated out of stainless steel and has a thickness of $0.5 \mathrm{~mm}$.

The influence of contamination on the thin film Seebeck coefficient was investigated by progressively increasing the background pressure before deposition. This was achieved by leaking atmospheric air into the vacuum chamber with a mass flow controller (MKS, Andover, MA, USA). After the background pressure is stabilized at a desired value, the sputter gas pressure is set by the introduction of argon gas (Argon 5.0 Praxair, Danbury, CT, USA) in the system. Finally the magnetron discharge is ignited and the target is sputtered at constant current $(0.6 \mathrm{~A})$. The power towards the magnetron is delivered by a Hüttinger 1500DC power supply (Ditzingen, Germany). The target-to-substrate distance and the argon pressure were kept constant during all depositions at $10 \mathrm{~cm}$ and 0.6 Pa. Except otherwise specified, all films, irrespective of the material, had a thickness of $350 \pm 30 \mathrm{~nm}$. The impurity-to-metal impingement flux ratio $\tau$ is used as a measure for the degree of contamination. This parameter is defined as the ratio between the impurity flux and the material flux impinging on the substrate surface, i.e., $\tau=F_{i} / F_{m}$. The material flux was calculated from the measured thickness (Taylor-Hobson profilometer, Leicester, UK), the deposition time, and the film density as measured by $\mathrm{x}$-ray reflectometry (Bruker D8). The impurity flux was calculated based on the Maxwell-Boltzmann distribution as presented in previous work [24].

The depositions for the experiment on the influence of the discharge current were performed under the same process conditions as the samples discussed above, i.e., target-to-substrate distance $10 \mathrm{~cm}$ and argon pressure $0.6 \mathrm{~Pa}$. The experiment was performed at two different background pressures. One series was deposited at a low background pressure $\left(3.50 \times 10^{-4} \mathrm{~Pa}\right)$ and one series at a high background pressure $\left(7.50 \times 10^{-3} \mathrm{~Pa}\right)$. The magnetron was sputtered in current-controlled way and the discharge current was varied from 0.6 to $0.1 \mathrm{~A}$. This was done in a random way in order to exclude any effects related to target erosion and/or chamber heating.

\section{Results}

\subsection{Impurities}

Figure 2 presents the results for the deviation of the thin film Seebeck coefficient from the bulk value, i.e., $|\Delta S|=\left|S_{B}-S_{F}\right|$, plotted as function of the impurity-to-metal impingement flux ratio $\tau$ for both copper (a) and constantan (b). In the low-impurity regime, i.e., $\tau \ll 1$, the Seebeck coefficient is not significantly affected by an increased background pressure. The average deviation from the bulk value in this low-impurity regime is represented by the dotted line for both copper $\left(1.9 \mu \mathrm{V} /{ }^{\circ} \mathrm{C}\right)$ and constantan $\left(2.61 \mu \mathrm{V} /{ }^{\circ} \mathrm{C}\right)$. In the high-impurity regime, i.e., $\tau \gg 1$, the Seebeck coefficient is strongly affected by an increasing degree of contamination. For both figures, error bars are included on a single marker but are valid for all data points in the respective plots. In order to simplify the rationale in Section 4.3, the striped lines in the figures represent the relation $|\Delta S| \sim \tau^{1 / 2}$ where the proportionality factor is fitted to coincide with the data in this high-impurity regime. This specific description of $|\Delta S|$ as function of the impurity-to-metal ratio $\tau$ embodies the thought of a relation between $|\Delta S|$ and the domain size $D$, more specifically $1 / D$. The idea for this relation originates from a previous work [24], where we elaborated a model that accounts for the experimental observation $D \sim \tau^{-1 / 2}$ in the high-impurity regime. The agreement between the experiment and the proposed relation $|\Delta S| \sim \tau^{1 / 2}$ 
is evaluated by means of a chi-squared test. For copper and constantan, we find a value $\chi^{2}=1.98$ (12 data points) and $\chi^{2}=3.21$ (9 data points) respectively. In both cases, the significance of the description is guaranteed within the $95 \%$ confidence interval. The full gray lines in Figure 2 represent the maximal deviation $|\Delta S|_{\max }$ which is found when $S_{F} \rightarrow 0$ and thus $|\Delta S|_{\max }=\left|S_{B}\right|$. We find for copper and constantan a value of $6.2 \mu \mathrm{V} /{ }^{\circ} \mathrm{C}$ and $40.1 \mu \mathrm{V} /{ }^{\circ} \mathrm{C}$ [25] respectively. Copper has a much smaller Seebeck coefficient in comparison to constantan. This hinders the electronic measurement and results in a high signal-to-noise ratio. As the observed trends are similar for both materials, we focus our research on constantan as the latter material has a higher Seebeck coefficient.

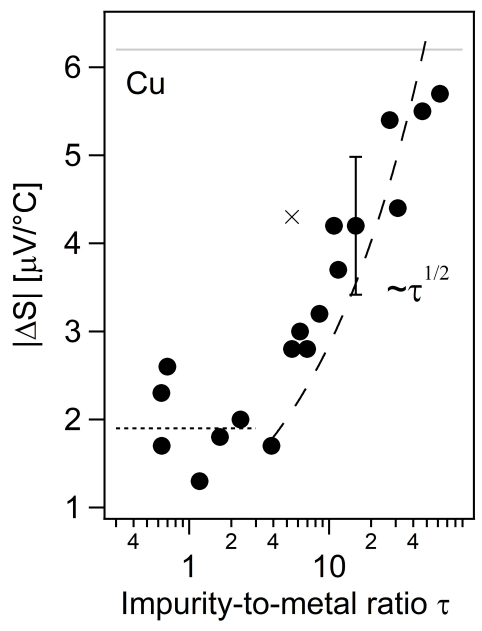

(a)

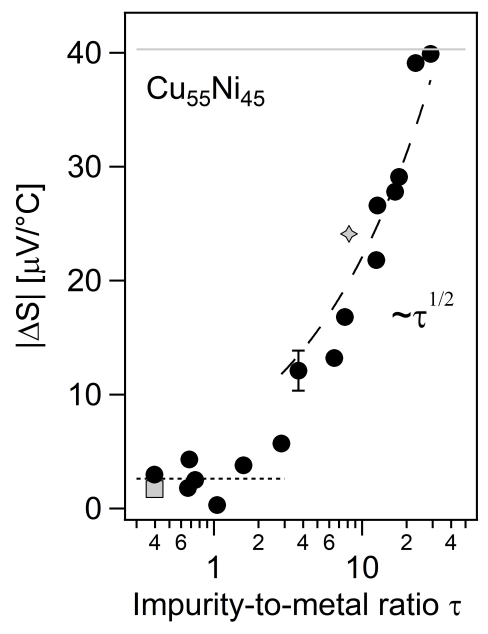

(b)

Figure 2. The deviation of the thin film Seebeck coefficient from the bulk value plotted as function of the impurity-to-metal impingement flux ratio for (a) copper and (b) constantan. The depositions are performed at a discharge current of $0.6 \mathrm{~A}$, an argon pressure of $0.6 \mathrm{~Pa}$ and a target-to-substrate distance of $10 \mathrm{~cm}$. The impurity-to-metal ratio was increased by leaking atmospheric impurities inside the vacuum chamber. The full gray lines in the figures indicate the maximal deviation $|\Delta S|_{\text {max }}$ for copper $\left(6.2 \mu \mathrm{V} /{ }^{\circ} \mathrm{C}\right)$ and constantan $\left(40.1 \mu \mathrm{V} /{ }^{\circ} \mathrm{C}\right)$. The dotted lines represent the average deviation from the bulk value in the low-impurity regime for copper $\left(1.9 \mu \mathrm{V} /{ }^{\circ} \mathrm{C}\right)$ and constantan $\left(2.61 \mu \mathrm{V} /{ }^{\circ} \mathrm{C}\right)$ and the striped lines represent the power law $|\Delta S| \sim \tau^{1 / 2}$. Error bars are included on a single marker but are valid for all data points in the respective plots. The data point represented by the cross marker (a) was excluded from the fit for the proportionality factor. The gray square and diamond markers (b) represent data points from the experiment discussed in Section 3.2.

\subsection{Discharge Current}

The results for the influence of the discharge current on the thin film Seebeck coefficient are presented in Figure 3. The square markers and diamond markers represent depositions performed at a background pressure of $3.50 \times 10^{-4} \mathrm{~Pa}$ and $7.50 \times 10^{-3} \mathrm{~Pa}$ respectively. The values for $|\Delta S|$ are significantly larger for the series deposited at a high background pressure compared to the series deposited at a low background pressure, an observation in accordance to the results discussed in Figure 2. As an illustration, the data points obtained in this experiment which corresponds to the same deposition conditions as the previous experiment, i.e., a discharge current of $0.6 \mathrm{~A}$, are represented by the gray (square and diamond) markers on the plot in Figure 2. For both series, the measurements indicate an increasing deviation of the film Seebeck coefficient from the bulk value with decreasing discharge current. 


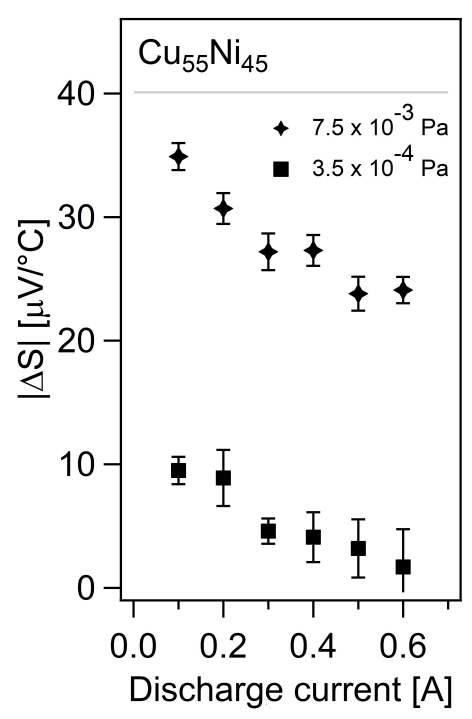

Figure 3. The deviation of the thin film Seebeck coefficient from the bulk value measured as function of the discharge current for 2 different background pressures: $3.50 \times 10^{-4} \mathrm{~Pa}$ (square markers) and $7.50 \times 10^{-3} \mathrm{~Pa}$ (diamond markers). The argon pressure was set to $0.6 \mathrm{~Pa}$ and the target-to-substrate distance was $10 \mathrm{~cm}$. The full gray line indicates the maximal deviation $|\Delta S|_{\max }$.

\subsection{Domain Size}

The domain sizes corresponding to the series deposited at varying discharge current at both low and high background pressure are plotted in Figure 4a. The domain size decreases with increasing background pressure and decreasing discharge current. As the discharge current is varied from $0.6 \mathrm{~A}$ up to $0.1 \mathrm{~A}$, the deposition speed was varied from $1.28 \mathrm{~nm} / \mathrm{s}$ up to $0.23 \mathrm{~nm} / \mathrm{s}$ respectively. Given the constant background pressure used within each series, the ratio of the impurity-to-metal impingement flux ratio $\tau$ thus increases with decreasing discharge current. The variation in background pressure between both series did not have any influence on the deposition speed. Figure $4 \mathrm{~b}$ is based on the same data for the domain size as in Figure 4a but presented in a normalized way and as function of the impurity-to-metal impingement flux ratio $\tau$. The data for the domain size have been normalized in order to allow a better comparison with the additional data set (gray circles) included in Figure $4 \mathrm{~b}$ which originates from a previous experiment [24]. The uppermost dotted line in the figures indicate the averaged domain size in this low-impurity regime. The domain size in the high-impurity regime $(\tau \gg 1)$ is strongly refined by the presence of the impurities during growth. Based on previous research [24], we expect a relation $D \sim \tau^{-1 / 2}$ in this regime. This relation is represented by the striped line and further consolidated by the gray markers taken from the previous work. In the low-impurity regime $(\tau \ll 1)$, the domain sizes are larger and less affected by an increase in impurity-to-metal impingement flux ratio. It must be noted here that in the determination of the domain sizes from the XRD data, we did not account for microstrain contributions. However, this analysis was carried out on the additional data set (gray circles Figure 4) from previous work, but no systematic variation in microstrain was observed.

Figure 5 summarizes the effect of background pressure (square markers vs. diamond markers) and discharge current (square markers and diamond markers) on $|\Delta S|$ described as function of the inverse of the domain size, i.e., $1 / D$. Additional data from the work of Barber et al. [26] are also included on the figure (round gray markers and unfilled blue markers). Whereas the data in the latter work are presented as $|\Delta S|$ as function of the FWHM, they are recalculated here using the Scherrer-equation in order to present them as function of $1 / D$. 


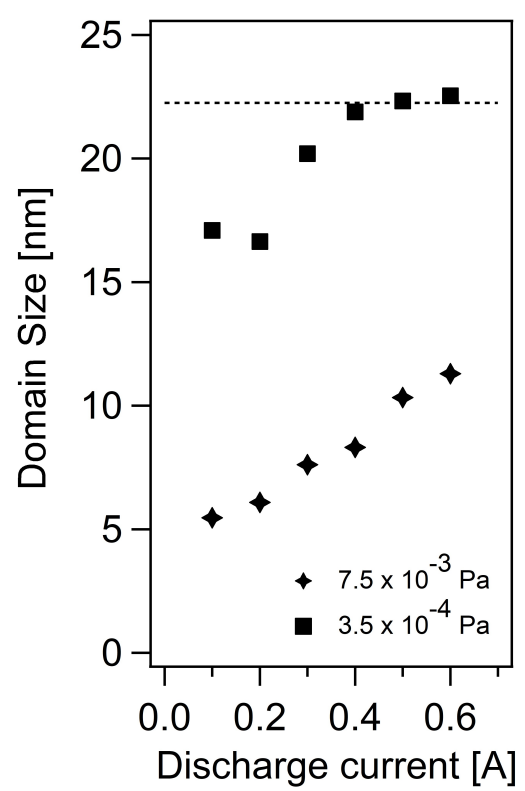

(a)

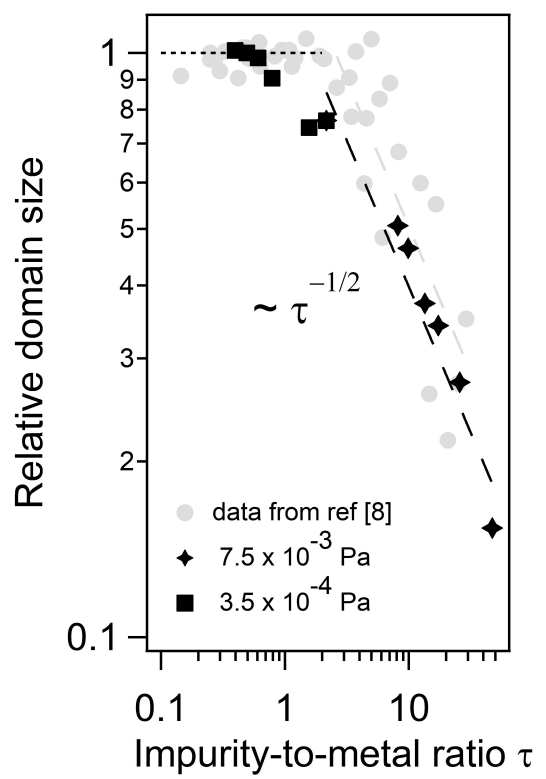

(b)

Figure 4. (a) Domain size as function of the discharge current; (b) Domain size presented as function of the impurity-to-metal impingement flux ratio. The square markers represent the deposition performed at low background pressure $\left(3.50 \times 10^{-4} \mathrm{~Pa}\right)$, the diamond markers represents the depositions performed at high background pressure $\left(7.50 \times 10^{-3} \mathrm{~Pa}\right)$. The dotted lines in the figures represents the average domain size in the low-impurity regime. The striped lines $(\mathbf{b})$ represent the relation $D \sim \tau^{-1 / 2}$.

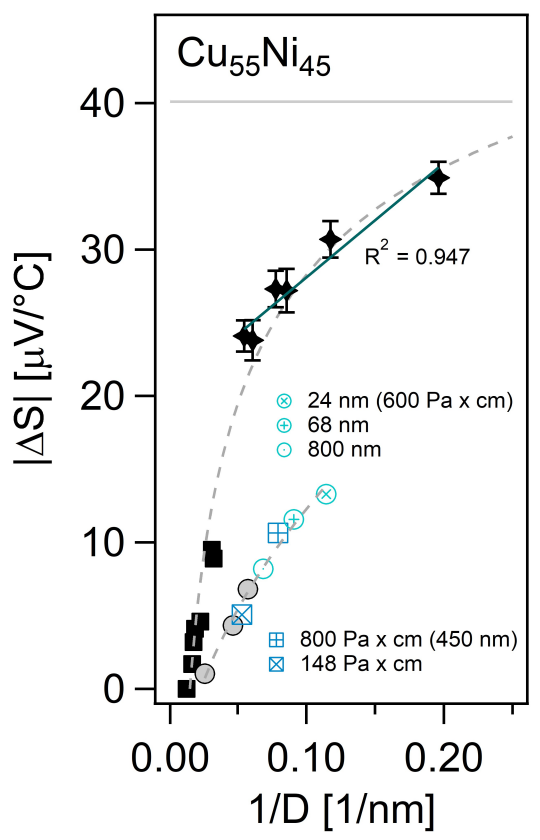

Figure 5. Deviation of the thin film Seebeck coefficient from the bulk value as function of the inverse of the domain size. The data derived from the work of Barber et al. [26] on sputter deposited constantan films are represented by the blue square unfilled markers (varying $P \times d$, constant thickness), the blue round unfilled markers (varying thickness, constant $P \times d$ ) and the round gray filled markers (both varying $P \times d$ and layer thickness). The full gray line represents the value for the maximal deviation $|\Delta S|_{\max }$. The gray dotted lines are a guide to the eye. 


\section{Discussion}

The work of Barber et al. [26] investigated the influence of the layer thickness and $P \times d$, i.e., the product of the sputter gas pressure $P$ times the target-to-substrate distance $d$, on the Seebeck coefficient of sputter deposited constantan films. The experiments showed that for a fixed $P \times d$-value, there is an increasing deviation $|\Delta S|$ from the bulk value as the film grows thinner. A similar observation was done by Gierczak et al. [27]. This is a classic observation of the size-effect. Inversely, at fixed layer thickness, $|\Delta S|$ increases with increasing $P \times d$-values. Only the data for which their respective domain size $D$ could be calculated are included in the plot in Figure 5. The influence of the layer thickness on the film Seebeck coefficient at fixed $P \times d$ is presented by the unfilled blue round markers, the influence of $P \times d$ at constant layer thickness is represented by the unfilled blue square markers. The round filled gray markers in Figure 5 represent combinations of a varying thickness together with a unspecified $P \times d$-value (thickness range $2000-90 \mathrm{~nm}$ ).

The main conclusion from the work of Barber is the direct correlation between the microstructure and the thin film Seebeck coefficient. The effect of the layer thickness was attributed to the morphological development as the film thickens. The dependency of the Seebeck coefficient on $P \times d$ is explained by thermalization effects affecting the microstructure. The role of the layer thickness is further discussed in Section 4.1, whereas the hypothesis of thermalization is confronted with our observations in Section 4.2.

\subsection{Role of Layer Thickness}

The electrical properties of a thin metal film deviate from the bulk as soon as one of the dimensions of the metal is restricted in the order of the electron mean free path length. The mean free path length of an electron in a metal is typically of the order of tens of nanometers [28], and is therefore easily restricted by the spatial dimensions of a thin film. The effects caused by this reduced mean free path are typically called "size-effects". For example, this is a major challenge in the field of microelectronics for contacting and connection paths in the sub-50 nm range. Over time, many models have been proposed in order to describe and understand the origin of these effects. The Fuchs-Sondheimer model [29] describes the increase in thin film resistivity from the perspective of an enhanced electron scattering caused by the decreased layer thickness. The model is described as function of $l_{0} / t$, i.e., the ratio of the electron mean free path $l_{0}$ to the layer thickness $t$, and a parameter $p$, representing the fraction of specular reflected electrons at the external film surfaces (e.g., substrate-film and film-air). The thinner the film, the more electron scattering events and thus the higher the electrical resistivity of the film, i.e., $\rho \sim 1 / t$. Later on, a similar model was proposed by Mayer [30] in order to describe the thermoelectric film properties. Analogously to the Fuchs and Sondheimer description for the electrical film resistivity, Mayer predicts the deviation of the thin film Seebeck coefficient from the bulk value to scale with the inverse of the layer thickness, i.e., $\left|S_{B}-S_{F}\right|=\left|\Delta S_{F}\right| \sim l_{0} / t$. Thus according to the Mayer theory, $\Delta S_{F}$ plotted as function of $1 / t$ yields a straight line. This behavior can indeed be verified by an abundant amount of experimental data present in the literature. For example, this is reported for sputtered $\mathrm{Ni}_{90} \mathrm{Cr}_{10}$ [31], for evaporated $\mathrm{Cu}[4,18,32,33], \mathrm{Ag}[18,34,35], \mathrm{Au}[35], \mathrm{Al}$ [36], Fe [3], Sn [21], Sb [37], Bi films [37-39], or electron-beam evaporated $\mathrm{Ni}$ [40].

Sputtered thin films grow in a columnar fashion with columns growing in a lateral direction with respect to the substrate and with a mean diameter $D_{i n}$, referred here to as the in-plane domain size [41,42]. In most cases, the in-plane domain size is measured by means of characterization techniques such as TEM, SEM, AFM or EBSD. When a sample is measured by means of XRD, one measure the degree of crystallographic coherence in the out-of-plane direction. The more (X-ray) interference events at consecutive crystallographic planes through the thickness of the sample, the sharper the peakwidths (FWHM) of the resulting diffractogram. Using the Scherrer-equation $\left(D_{\text {out }} \sim(F W H M)^{-1}\right)$, sharp peaks with a small FWHM yield large (out-of-plane) domains $D_{\text {out }}$. For sputtered as-deposited films, the in-plane domain size $D_{i n}$ measured by TEM is very similar to the out-of-plane domain size $D_{\text {out }}$ as measured by XRD [43]. Therefore, in what follows, we will use the 
out-of-plane domain size as measured by XRD as a representative measure for the in-plane domain size and both in-plane and out-of-plane are simply referred to as the domain size $D$.

For thin films grown by physical vapor deposition in general, it is an observation that the domain size increases with the film thickness, i.e., $D \sim t^{\chi}$, with $\chi$ a value between $1 / 3$ and $1 / 2$ [44-48], depending on the growth mode, substrate temperature and more. This can be verified with experimental data presented in the literature for sputtered $\mathrm{Cu}$ [49-51], $\mathrm{Ag}$ [52,53], Mo [42], Fe [54], $\mathrm{FeCo}$ [55], $\mathrm{CuNi}$ [56], $\mathrm{NiCr}$ [56] and $\mathrm{Sb}_{2} \mathrm{Te}_{3}$ [57], or for evaporated $\mathrm{Ni}_{80} \mathrm{Fe}_{20}$ (permalloy) films [58] or ion beam-evaporated $\mathrm{Ni}$ film [40]. Variations in film thickness are thus linked with variations in the domain size. The thinner the film, the smaller the resulting domains. Typically, for sputtered as-deposited thin films, $D<t[42,51-57]$. Therefore, the size-depend effects observed for films with decreasing thickness can thus either be attributed to variations in $D$ or $t$ [59]. Indeed, when electrons flow through a thin film under influence of an electric field (resistivity) or temperature difference (thermoelectricity), the net flow is parallel to the substrate and film surface and thus perpendicular to the growth direction (out-of-plane) of the grains. This, in combination with the observations that $D<t$, makes it very likely that a much higher contribution of electron scattering at grain boundaries is to be expected for sputtered as-deposited films, rather than the scattering contribution from the external film surfaces as predicted by the Fuchs-Sondheimer or Mayer theory. The idea of an additional grain-boundary scattering contribution to the Fuchs-Sondheimer scattering model was already implemented under the Mayadas-Shatzkes model [41] for the description of the thin film resistivity. The shortcomings of the Fuchs-Sondheimer model to describe experimental data and the many experimental validations of the MS-model proved the dominant character of grain-boundary scattering [41,50,60,61]. Later on, Deschacht et al. [62] elaborated a similar analytical model for the description of the thermoelectric power of polycrystalline semimetal films taking into account the effects of grain boundaries. The strong contribution grain-boundary scattering could have on the Seebeck coefficient was also already hinted by other authors $[26,40,63]$. However, to our knowledge, elaborated experimental studies on the Seebeck coefficient of sputtered thin films, their relation to deposition conditions [64] and proof for the dominant character of the domain size on the Seebeck coefficient of (metallic) thin films [26] remains very limited. Based on the above discussion and in agreement with the latter work of Barber et al. [26], we conclude that the microstructural development during film growth can account for the observed variations in the film Seebeck coefficient as function of the layer thickness. In what follows, the layer thickness is kept constant $(350 \mathrm{~nm})$ for all depositions in order to exclude this influence from the discussion.

\subsection{Process Parameters}

The main effect of varying deposition conditions such as the discharge current $I$ or the argon pressure $P$ and the target-to-substrate distance $d$, is a variation in energy flux and/or material flux arriving on the substrate. As both the energy flux and the material flux are system-dependent parameters, i.e., not easily transferable towards other vacuum systems, we prefer to project them on system-objective parameters such as the energy per arriving adatom (EPA) and the impurity-to-metal impingement flux ratio $\tau$. The EPA has different energetic contributions such as a contribution from the kinetic energy of the sputtered particles or reflected neutrals, from electronic contributions, and from plasma radiation. Whereas the latter two contributions are mainly affected by the target-to-substrate distance $d$, the former two are mainly affected by $P \times d$, i.e., the product of the argon pressure and the target-to-substrate distance. As discussed in the work of Z. Barber et al. [26], $P \times d$ is a measure for the degree of thermalization. As thermalized adatoms have a restricted mobility on the substrate surface, large $P \times d$-values result in smaller grains.

In contrast to $P \times d$, the EPA remains mainly indifferent under a variation in discharge current. This because when the discharge current is decreased, both the decrease in material flux arriving on the substrate as well as the total power dissipated in the system approximately scale in a linear way [56]. The EPA is thus not significantly affected by a variation in the discharge current. The experimental 
result presented in Figure 3 is therefore not compatible with the idea of a variation in film Seebeck coefficient caused by thermalization effects. However, as the deposition speed drops with a decreasing discharge current, this causes the impurity-to-metal ratio $\tau$ to increase. It is shown in Figure 4 (right) that this latter effect is responsible for the decrease of the domain size with decreasing discharge current. Analogously, the impurity-to-metal ratio $\tau$ increases with increasing $P \times d$ as $P \times d$, and especially $d$, affects the deposition speed [56]. It can also be verified in the literature that for an increasing value for $P \times d$, the domain size decreases [65-67]. Our observations therefore indicate that a more consistent description for the effect of the discharge current on the thin film Seebeck coefficient (as presented in Figure 3) can be given from the perspective of refined grains - in coherence with the results reported in the work of Barber - but caused by an increased impurity-to-metal impingement flux $\tau$ rather than caused by thermalization effects. Furthermore, the $P \times d$-values used in the latter work, i.e., in the range of 150-800 Pa mm, are very high in comparison to the $P \times d$-value used in this work, i.e., $60 \mathrm{~Pa} \mathrm{~mm}$. The EPA values in the work of Barber are thus expected to be much lower than in this work. According to SiMTra simulations, i.e., a kinetic Monte Carlo code for the simulation of metal transport through the gas phase [68], the energy of the sputtered particles in the range of 150-800 Pa mm is estimated betzeen $1.5 \mathrm{eV}$ and $3 / 2 k T(T$ at $300 \mathrm{~K}$ ) respectively and around $6 \mathrm{eV}$ for the $P \times d$-value (60 Pa $\mathrm{mm}$ ) used in this work. The simulations indicate fully thermalized sputtered particles, i.e., $E=3 / 2 k T$, at a $P \times d$-value close to $400 \mathrm{~Pa} \mathrm{~mm}$. Therefore, it seems less likely that the variations in $|\Delta S|$ as function of $P \times d$ (see Figure 2 in [26]) could be attributed to thermalization effects, especially for the data discussed where $P \times d>400 \mathrm{~Pa} \mathrm{~mm}$. Of course, other contributions could still contribute to the energy balance for the adatoms, although for this material-sputter gas combination, the amount of reflected neutrals is expected to be low $[69,70]$ and the electronic and radiative contributions rather scale as function of $d$ and not as $P \times d$ which is the expected dependency based on scattering events for massive particles. However, in order to demonstrate our point-of-view with respect to the importance of impurity incorporation, in the following section, both the layer thickness and the EPA are kept constant to eliminate thermalization effects and solely the impurity-to-metal ratio $\tau$ is altered.

\subsection{Impurities}

As already discussed in previous work [24], atmospheric gas impurities present in the vacuum chamber during film growth can act as grain refiners. In general, in the high-impurity regime $(\tau \gg 1)$, the average domain size $D$ decreases in a material-independent way with an increasing impurity-to-metal impingement flux ratio $\tau$ according to $D \sim \tau^{-1 / 2}$ [24]. This effect was experimentally verified in Figure 4 (right). The relation embodies the thought of a nucleation-dominated growth model. When the spatial distribution of impinging impurities on the surface is of the order of the characteristic length of the diffussing adatoms, the impurities act as active nucleation centra and restrict the adatom diffusion with refined grains as a consequence. The results presented in Figure 2 showed how the film Seebeck coefficient was strongly affected in this high-impurity regime. The similarity in behavior between the domain size $D \sim \tau^{-1 / 2}$ and the Seebeck coefficient as function of the impurity-to-metal ratio $|\Delta S| \sim \tau^{1 / 2}$ (see Figure 2) further empowers the perspective to describe the Seebeck coefficient as a film property determined by the domain size. Based on our assumptions, an approximate linear relationship $|\Delta S| \sim 1 / D$ can thus be expected in this high-impurity regime. As presented in Figure 5 (diamond markers), this prediction can be fairly well validated. Furthermore, in analogy to the work of Liu et al. [71] where the deviation in film Seebeck coefficient due to the size-effect is employed for manufacturing single-metal thermocouples, the observation of a decreased thin film Seebeck coefficient as function of $\tau$ could be exploited for creating thermocouples where both legs consist of the same material but are deposited at a different $\tau$-value.

\section{Conclusions}

The effect of impurities, layer thickness and deposition conditions such as discharge current and $P \times d$ on the thin film Seebeck coefficient is discussed. It was emphasized here how a variation in 
each of these deposition variables can affect the domain size. In view of the diversity of experimental variations which could be summarized as function of the domain size, more specifically, $1 / D$, it is justified to conclude that rather than the layer thickness, the domain size has a key role in the deviation of the thin film Seebeck coefficient from the bulk value. Therefore, the influence of deposition conditions on the Seebeck coefficient can be evaluated by understanding their effect on the domain size. This conclusion is mainly in coherence with a the work on sputter-deposited constantan by Barber et al. [26], i.e., there is a direct correlation between the microstructure and the Seebeck coefficient, but where our results indicate that the origin of the grain refinement can be attributed to an increased impurity incorporation during growth rather than to thermalization effects. Of course, it is not straightforward to discriminate between the active mechanisms as an increase in the degree of thermalization and a decrease in the deposition rate, and thus an increase in impurity-to-metal impingement flux ratio $\tau$, are unambiguously linked through an increasing target-to-substrate distance $d$. Although the data of the experiments performed in this work and in the work of Barber are quantitatively shifted in $|\Delta S|$-which we believe can be attributed to experimental differences-this does not compromise on the generality of the discussion as the qualitative response of the Seebeck coefficient to the variations in domain size is very comparable.

Author Contributions: Conceptualization, F.G.C. and D.D.; methodology, F.G.C.; software, F.G.C.; validation, F.G.C.; formal analysis, F.G.C. and D.D.; investigation, F.G.C.; resources, F.G.C.; data curation, F.G.C.; writing-original draft preparation, F.G.C.; writing-review and editing, D.D.; visualization, F.G.C.; supervision, D.D.; project administration, D.D.; funding acquisition, D.D.

Funding: This research was funded by Ghent University through the GOA-ENCLOSE project (BOF15/GOA/007).

Conflicts of Interest: The authors declare no conflict of interest.

\section{Abbreviations}

$\begin{array}{ll}\text { EPA } & \text { Energy per arriving adatom } \\ P & \text { Argon Pressure } \\ d & \text { Target-to-substrate distance } \\ D & \text { Domain Size } \\ t & \text { Layer thickness } \\ \text { FWHM } & \text { Full width at half maximum } \\ \text { RF } & \text { Radio-frequent } \\ \text { DC } & \text { Direct-current } \\ \text { RCA } & \text { Radio Corporation of America } \\ \text { MS } & \text { Mayadas-Shatzkes } \\ \text { PID } & \text { Proportional-integral-derivative } \\ \text { XRD } & \text { X-ray diffraction } \\ \text { TEM } & \text { Transmission electron microscope } \\ \text { AFM } & \text { Atomic force microscopy } \\ \text { EBSD } & \text { Electron backscatter diffraction }\end{array}$

\section{References}

1. Laugier, $\mathrm{M}$. The construction and use of thin film thermocouples for the measurement of surface temperature: Applications to substrate temperature determination and thermal bending of a cantilevered plate during film deposition. Thin Solid Films 1980, 67, 163-170. [CrossRef]

2. Kreider, K.G. Sputtered high temperature thin film thermocouples. J. Vac. Sci. Technol. A Vac. Surf. Films 1993, 11, 1401-1405. [CrossRef]

3. Scarioni, L.; Castro, E. Thermoelectric power in thin film Fe-CuNi alloy (type-J) couples. J. Appl. Phys. 2000, 87, 4337-4339. [CrossRef]

4. Chopra, K.; Bahl, S.; Randlett, M. Thermopower in thin-film copper-Constantan couples. J. Appl. Phys. 1968, 39, 1525-1528. [CrossRef] 
5. Guo, H.; Jiang, J.Y.; Liu, J.X.; Nie, Z.H.; Ye, F.; Ma, C.F. Fabrication and Calibration of Cu-Ni Thin Film Thermocouples. Adv. Mater. Res. 2012, 512-515, 2068-2071. [CrossRef]

6. Sadat, S.; Tan, A.; Chua, Y.J.; Reddy, P. Nanoscale thermometry using point contact thermocouples. Nano Lett. 2010, 10, 2613-2617. [CrossRef] [PubMed]

7. Kim, K.; Jeong, W.; Lee, W.; Reddy, P. Ultra-high vacuum scanning thermal microscopy for nanometer resolution quantitative thermometry. Acs Nano 2012, 6, 4248-4257. [CrossRef]

8. Kim, K.; Song, B.; Fernández-Hurtado, V.; Lee, W.; Jeong, W.; Cui, L.; Thompson, D.; Feist, J.; Reid, M.H.; García-Vidal, F.J.; et al. Radiative heat transfer in the extreme near field. Nature 2015, 528, 387. [CrossRef]

9. Liu, H.; Sun, W.; Xiang, A.; Shi, T.; Chen, Q.; Xu, S. Towards on-chip time-resolved thermal mapping with micro-/nanosensor arrays. Nanoscale Res. Lett. 2012, 7, 484. [CrossRef]

10. Li, G.; Wang, Z.; Mao, X.; Zhang, Y.; Huo, X.; Liu, H.; Xu, S. Real-time two-dimensional mapping of relative local surface temperatures with a thin-film sensor array. Sensors 2016, 16, 977. [CrossRef]

11. Guk, E.; Ranaweera, M.; Venkatesan, V.; Kim, J.S. Performance and durability of thin film thermocouple array on a porous electrode. Sensors 2016, 16, 1329. [CrossRef] [PubMed]

12. Meredith, R.D.; Wrbanek, J.D.; Fralick, G.C.; Greer, L.C.; Hunter, G.W.; Chen, L. Design and operation of a fast, thin-film thermocouple probe on a turbine engine. In Proceedings of the 50th AIAA/ASME/SAE/ASEE Joint Propulsion Conference, Cleveland, OH, USA, 28-30 July 2014; p. 3923.

13. Zeng, Q.Y.; Hong, T.; Chen, L.; Cui, Y.X. Magnetron sputtering of NiCr/NiSi thin-film thermocouple sensor for temperature measurement when machining chemical explosive material. Key Eng. Mater. 2011, 467, 134-139. [CrossRef]

14. Geballe, T.; Hull, G. Seebeck effect in silicon. Phys. Rev. 1955, 98, 940. [CrossRef]

15. Gayner, C.; Kar, K.K. Recent advances in thermoelectric materials. Prog. Mater. Sci. 2016, 83, 330-382. [CrossRef]

16. Marshall, R.; Atlas, L.; Putner, T. The preparation and performance of thin film thermocouples. J. Sci. Instrum. 1966, 43, 144. [CrossRef]

17. Lin, S.F.; Leonard, W.F. Thermoelectric power of thin gold films. J. Appl. Phys. 1971, 42, 3634-3639. [CrossRef]

18. Yu, H.Y.; Leonard, W.F. Thermoelectric power of thin silver films. J. Appl. Phys. 1973, 44, 5324-5327. [CrossRef]

19. Leonard, W.F.; Yu, H. Thermoelectric power of thin copper films. J. Appl. Phys. 1973, 44, 5320-5323. [CrossRef]

20. Angadi, M.A.; Ashrit, P.V. Thermoelectric effect in ytterbium and samarium films. J. Phys. D Appl. Phys. 1981, 14, L125-L128. [CrossRef]

21. Angadi, M.; Udachan, L. Thermoelectric power measurements in thin tin films. J. Phys. D Appl. Phys. 1981, 14, L103. [CrossRef]

22. Angadi, M.A.; Shivaprasad, S.M. Thermoelectric power measurements in thin palladium films. J. Mater. Sci. Lett. 1982, 1, 65-66. [CrossRef]

23. Angadi, M.A.; Udachan, L.A. Thermopower measurements in chromium films. J. Mater. Sci. Lett. 1982, 1, 539-541. [CrossRef]

24. Cougnon, F.; Dulmaa, A.; Dedoncker, R.; Galbadrakh, R.; Depla, D. Impurity dominated thin film growth. Appl. Phys. Lett. 2018, 112, 221903. [CrossRef]

25. Guan, A.; Wang, H.; Jin, H.; Chu, W.; Guo, Y.; Lu, G. An experimental apparatus for simultaneously measuring Seebeck coefficient and electrical resistivity from $100 \mathrm{~K}$ to 600 K. Rev. Sci. Instrum. 2013, 84, 043903. [CrossRef]

26. Barber, Z.; Somekh, R. Magnetron sputtering of Cu55Ni45. Vacuum 1984, 34, 991-994. [CrossRef]

27. Gierczak, M.; Prażmowska-Czajka, J.; Dziedzic, A. Thermoelectric mixed thick-/thin film microgenerators based on constantan/silver. Materials 2018, 11, 115. [CrossRef]

28. Gall, D. Electron mean free path in elemental metals. J. Appl. Phys. 2016, 119, 085101. [CrossRef]

29. Sondheimer, E.H. The mean free path of electrons in metals. Adv. Phys. 1952, 1, 1-42. [CrossRef]

30. Mayer, H. Recent developments in conduction phenomena in thin metal films. In Structure and Properties of Thin Films; Neugebauer, C.A., Newkirk, J.W., Eds; Wiley: New York, NY, USA, 1959; p. 225.

31. Zhang, X.; Choi, H.; Datta, A.; Li, X. Design, fabrication and characterization of metal embedded thin film thermocouples with various film thicknesses and junction sizes. J. Micromech. Microeng. 2006, 16, 900. [CrossRef] 
32. Rao, V.N.; Mohan, S.; Reddy, P.J. Electrical resistivity, TCR and thermoelectric power of annealed thin copper films. J. Phys. D Appl. Phys. 1976, 9, 89. [CrossRef]

33. Thakoor, A.; Suri, R.; Suri, S.; Chopra, K. Electron transport properties of copper films. II. Thermoelectric power. J. Appl. Phys. 1975, 46, 4777-4783. [CrossRef]

34. Rao, V.N.; Mohan, S.; Reddy, P.J. The size effect in the thermoelectric power of silver films. Thin Solid Films 1977, 42, 283-289. [CrossRef]

35. Hubin, M.; Gouault, J. Resistivity and thermoelectric power between $-100{ }^{\circ} \mathrm{C}$ and $+100{ }^{\circ} \mathrm{C}$ of gold and silver thin films formed and studied in ultrahigh vacuum. Thin Solid Films 1974, 24, 311-331. [CrossRef]

36. De, D.; Bandyopadhyay, S.K.; Chaudhuri, S.; Pal, A.K. Thermoelectric power of aluminum films. J. Appl. Phys. 1983, 54, 4022-4027. [CrossRef]

37. Boyer, A.; Cisse, E. Properties of thin film thermoelectric materials: Application to sensors using the Seebeck effect. Mater. Sci. Eng. B 1992, 13, 103-111. [CrossRef]

38. Das, V.D.; Soundararajan, N. Size and temperature effects on the Seebeck coefficient of thin bismuth films. Phys. Rev. B 1987, 35, 5990. [CrossRef]

39. Mikolajczak, P.; Piasek, W.; Subotowicz, M. Thermoelectric power in bismuth thin films. Phys. Status Solidi A 1974, 25, 619-628. [CrossRef]

40. Bourque-Viens, A.; Aimez, V.; Taberner, A.; Nielsen, P.; Charette, P.G. Modelling and experimental validation of thin-film effects in thermopile-based microscale calorimeters. Sens. Actuators A Phys. 2009, 150, 199-206. [CrossRef]

41. Mayadas, A.; Shatzkes, M. Electrical-resistivity model for polycrystalline films: The case of arbitrary reflection at external surfaces. Phys. Rev. B 1970, 1, 1382. [CrossRef]

42. Hofer, A.; Schlacher, J.; Keckes, J.; Winkler, J.; Mitterer, C. Sputtered molybdenum films: Structure and property evolution with film thickness. Vacuum 2014, 99, 149-152. [CrossRef]

43. Braeckman, B.; Misják, F.; Radnóczi, G.; Caplovicova, M.; Djemia, P.; Tetard, F.; Belliard, L.; Depla, D. The nanostructure and mechanical properties of nanocomposite $\mathrm{Nb}_{x}$-CoCrCuFeNi thin films. Scr. Mater. 2017, 139, 155-158. [CrossRef]

44. Xin, Z.; Xiao-Hui, S.; Dian-Lin, Z. Thickness dependence of grain size and surface roughness for dc magnetron sputtered Au films. Chin. Phys. B 2010, 19, 086802. [CrossRef]

45. Srolovitz, D.J.; Battaile, C.C.; Li, X.; Butler, J.E. Simulation of faceted film growth in two-dimensions: Microstructure, morphology and texture. Acta Mater. 1999, 47, 2269-2281.

46. Song, X.; Liu, G. Computer simulation of normal grain growth in polycrystalline thin films. J. Mater. Sci. 1999, 34, 2433-2436. [CrossRef]

47. Thijssen, J. Simulations of polycrystalline growth in 2+1 dimensions. Phys. Rev. B 1995, 51, 1985. [CrossRef]

48. Van der Drift, A. Evolutionary selection, a principle governing growth orientation in vapour-deposited layers. Philips Res. Rep. 1967, 22, 267.

49. Barmak, K.; Darbal, A.; Ganesh, K.J.; Ferreira, P.J.; Rickman, J.M.; Sun, T.; Yao, B.; Warren, A.P.; Coffey, K.R. Surface and grain boundary scattering in nanometric $\mathrm{Cu}$ thin films: A quantitative analysis including twin boundaries. J. Vac. Sci. Technol. A Vac. Surf. Films 2014, 32, 061503. [CrossRef]

50. Sun, T.; Yao, B.; Warren, A.P.; Barmak, K.; Toney, M.F.; Peale, R.E.; Coffey, K.R. Dominant role of grain boundary scattering in the resistivity of nanometric Cu films. Phys. Rev. B 2009, 79, 041402. [CrossRef]

51. Zhang, X.; Misra, A. Residual stresses in sputter-deposited copper/330 stainless steel multilayers. J. Appl. Phys. 2004, 96, 7173-7178. [CrossRef]

52. Yu, S.; Li, L.; Lyu, X.; Zhang, W. Preparation and investigation of nano-thick FTO/Ag/FTO multilayer transparent electrodes with high figure of merit. Sci. Rep. 2016, 6, 20399. [CrossRef]

53. Ding, G.; Clavero, C. Silver-based low-emissivity coating technology for energy-saving window applications. In Modern Technologies for Creating the Thin-film Systems and Coatings; Nikitenkov, N., Ed.; InTechOpen: London, UK, 2017; pp. 409-431.

54. Shamsutdinov, N.; Sloof, W.; Böttger, A. A method for the experimental determination of surface photoemission core-level shifts for 3d transition metals. J. Appl. Phys. 2005, 98, 014908. [CrossRef]

55. Krist, T.; Teichert, A.; Meltchakov, E.; Vidal, V.; Zoethout, E.; Müllender, S.; Bijkerk, F. Stress reduction in multilayers used for X-Ray and neutron optics. In Modern Developments in X-Ray and Neutron Optics; Erko, A., Idir, M., Eds.; Springer: Heidelberg, Germany, 2008; pp. 371-388. 
56. Cougnon, F.; Schramm, I.; Depla, D. On the electrical properties of sputter deposited thin films: The role of energy and impurity flux. Thin Solid Films 2019, submitted for publication.

57. Wanarattikan, P.; Jitthammapirom, P.; Sakdanuphab, R.; Sakulkalavek, A. Effect of grain size and film thickness on the thermoelectric properties of flexible $\mathrm{Sb}_{2} \mathrm{Te}_{3}$ thin films. Adv. Mater. Sci. Eng. 2019, 2019, 6954918. [CrossRef]

58. Mayadas, A.F.; Janak, J.F.; Gangulee, A. Resistivity of Permalloy thin films. J. Appl. Phys. 1974, 45, 2780. [CrossRef]

59. Sun, T.; Yao, B.; Warren, A.P.; Barmak, K.; Toney, M.F.; Peale, R.E.; Coffey, K.R. Surface and grain-boundary scattering in nanometric Cu films. Phys. Rev. B 2010, 81, 155454. [CrossRef]

60. Zhang, W.; Brongersma, S.; Heylen, N.; Beyer, G.; Vandervorst, W.; Maex, K. Geometry effect on impurity incorporation and grain growth in narrow copper lines. J. Electrochem. Soc. 2005, 152, C832-C837. [CrossRef]

61. Birkett, M.; Penlington, R. Electrical resistivity of CuAlMo thin films grown at room temperature by dc magnetron sputtering. Mater. Res. Express 2016, 3, 075021. [CrossRef]

62. Deschacht, D.; Boyer, A.; Groubert, E. The thermoelectric power of polycrystalline semimetal films. Phys. Status Solidi A 1982, 71, K205-K209. [CrossRef]

63. Beensh-Marchwicka, G.; Osadnik, S.; Prociów, E.; Mielcarek, W. Structure and morphology of Ge(Au) sputtered films with useful Seebeck effect. Vacuum 1998, 50, 207-210. [CrossRef]

64. Arab Pour Yazdi, M.; Martin, N.; Petitot, C.; Neffaa, K.; Palmino, F.; Cherioux, F.; Billard, A. Influence of sputtering parameters on structural, electrical and thermoelectric properties of $\mathrm{Mg}-\mathrm{Si}$ coatings. Coatings 2018, 8, 380. [CrossRef]

65. Gordillo, G.; Mesa, F.; Calderón, C. Electrical and morphological properties of low resistivity Mo thin films prepared by magnetron sputtering. Braz. J. Phys. 2006, 36, 982-985. [CrossRef]

66. Chan, K.; Teo, B. Effect of Ar pressure on grain size of magnetron sputter-deposited Cu thin films. IET Sci. Meas. Technol. 2007, 1, 87-90. [CrossRef]

67. Qiu, H.; Wang, F.; Wu, P.; Pan, L.; Tian, Y. Structural and electrical properties of Cu films deposited on glass by DC magnetron sputtering. Vacuum 2002, 66, 447-452. [CrossRef]

68. Van Aeken, K.; Mahieu, S.; Depla, D. The metal flux from a rotating cylindrical magnetron: A Monte Carlo simulation. J. Phys. D Appl. Phys. 2008, 41, 205307. [CrossRef]

69. Hoffman, D.; Thornton, J.A. Internal stresses in $\mathrm{Cr}, \mathrm{Mo}$, Ta, and Pt films deposited by sputtering from a planar magnetron source. J. Vac. Sci. Technol. 1982, 20, 355-358. [CrossRef]

70. Depla, D.; Mahieu, S. Reactive Sputter Deposition; Springer: Heidelberg, Germany, 2008.

71. Liu, H.; Sun, W.; Xu, S. An extremely simple thermocouple made of a single layer of metal. Adv. Mater. 2012, 24, 3275-3279. [CrossRef]

(C) 2019 by the authors. Licensee MDPI, Basel, Switzerland. This article is an open access article distributed under the terms and conditions of the Creative Commons Attribution (CC BY) license (http://creativecommons.org/licenses/by/4.0/). 